\title{
Assessing the relationship between admission glucose levels, subsequent length of hospital stay, readmission and mortality
}

\author{
NR Evans and KK Dhatariya
}

\begin{abstract}
This study aimed to investigate relationships between dysglycaemia and length of hospital stay, short-term mortality and readmission in an unselected population in an acute medical unit (AMU). The rate of follow up in non-diabetic individuals with hyperglycaemia was also measured. We analysed data from all 1,502 patients admitted through our AMU in February 2010 to assess blood glucose levels on admission, length of stay, 28-day readmissions and mortality, and to determine whether blood glucose $\geqslant 11.1 \mathrm{mmol} / \mathrm{l}$ on admission in non-diabetic individuals was followed up. In total, blood glucose was measured on admission for 893 patients. Mean length of stay was 8.8 (standard deviation 11.9) days, for patients with blood glucose $<6.5 \mathrm{mmol} / \mathrm{l}$ on admission; 11.3 (13.6) days, for 6.5-7 mmol/l; 10.2 (14.5) days, for 7.1-9 mmol/l; 10.6 (14.9) days, for $9.1-11 \mathrm{mmol} / \mathrm{l}$; 12 (18.4) days, for $11.1-20 \mathrm{mmol} / \mathrm{l}$ and 9.1 (11.2) days, for $>20.1 \mathrm{mmol} / \mathrm{l}$. Length of stay for patients with blood glucose $>6.5 \mathrm{mmol} / \mathrm{l}$ on admission was significantly longer $(p=0.002)$. The 28 -day readmission rates were $6.4 \%, 6 \%$, $9.7 \%, 12.5 \%, 10 \%$ and $15 \%$, respectively, and 28 -day death rates were $4.8 \%, 6 \%, 5.8 \%, 17.2 \%, 17.1 \%$ and $6.1 \%$, respectively. Overall, $51.4 \%$ of non-diabetic individuals with blood glucose $>11.1 \mathrm{mmol} / \mathrm{l}$ on admission were followed up. The study showed that blood glucose $>6.5 \mathrm{mmol} / \mathrm{l}$ on admission is associated with significantly longer length of stay. Hyperglycaemia was associated with increased 28-day mortality and readmissions, and is frequently underinvestigated.
\end{abstract}

\section{KEY WORDS: dysglycaemia, glucose, length of stay}

\section{Introduction}

High levels of blood glucose have been shown to be strongly associated with increased length of hospital admission and poorer short-term prognosis, regardless of the presence of diabetes mellitus. ${ }^{1-4}$ Most previous research in this area has focused on the relationship between dysglycaemia and specific conditions $^{2-4}$ on admission to specialist care facilities (eg different intensive care units). ${ }^{5}$ In this study, we investigate the relationship between dysglycaemia and length of hospital stay, shortterm (28-day) mortality and readmissions in an unselected

NR Evans, trainee in medicine and KK Dhatariya, consultant in diabetes and endocrinology

Elsie Bertram Diabetes Centre, Norfolk and Norwich University Hospitals NHS Foundation Trust, Colney Lane, Norwich acute medical unit (AMU) population. In addition, this study investigates the rate of follow up in individuals found to have hyperglycaemia on admission who did not have an existing diagnosis of diabetes. Other centres have highlighted the poor rate of follow up in such individuals. ${ }^{6}$

The Norfolk and Norwich University Hospital is a large teaching hospital with a geographical catchment area of about 2,000 square miles and a population of about 600,000 people. The Elsie Bertram Diabetes Centre based at the hospital provides care to nearly 4,000 registered patients, largely those with complex and/or insulin-treated diabetes. The centre has two whole-time equivalent inpatient diabetes specialist nurses and a network of four whole-time equivalent nurse facilitators, who provide direct practice support to primary care. Patients are admitted to the AMU through direct referral by their GP or through referral from the emergency department at the hospital.

\section{Patients and methods}

The medical and computer records for each emergency medical admission in a non-selected population admitted through the AMU during February 2010 were reviewed retrospectively for the length of hospital stay and short-term (28-day) mortality and readmission rates. Blood glucose on admission was measured using venous samples taken at the time of first physician review in the emergency department (prior to referral to the $A M U)$ or in the AMU if the patient was referred there directly and prior to any treatment.

Following admission to the AMU, patients were triaged to one of 11 different medical specialties: medicine for the elderly, cardiology, respiratory, nephrology, gastroenterology, endocrinology, neurology, dermatology, haematology, oncology and general medicine. In our trust, patients triaged to 'general medicine' are those individuals who do not fall under any specific category - eg overdose, pulmonary embolism, pyrexia of unknown origin and so forth. These patients are triages to either 'general medicine - renal' or 'general medicine - endocrinology'. The split is dependent on how many people have been triaged to each specialty already that day in an attempt to divide them equally between the two specialties.

For the purposes of comparing length of hospital stay and 28 -day readmission and mortality, patients were divided into six groups based on their blood glucose on admission: $<6.5$ $\mathrm{mmol} / \mathrm{l}, \quad 6.5-7 \mathrm{mmol} / \mathrm{l}, \quad 7.1-9 \mathrm{mmol} / \mathrm{l}, 9.1-11 \mathrm{mmol} / \mathrm{l}$, 11.1-20 mmol/l and $>20 \mathrm{mmol} / \mathrm{l}$. The rate of follow up of individuals with random blood glucose $\geqslant 11.1 \mathrm{mmol} / \mathrm{l}$ on 
admission was compared between the different triaged medical specialties. For the purposes of this study, follow up was counted as fasting blood glucose within 28 days or review by a diabetes specialist (diabetes specialist nurse, diabetes specialist registrar or consultant).

\section{Results}

In total, 1,502 individuals were admitted during the study period and accounted for 14,202 patient-days. Of these, 240 (16\%) had an established diagnosis of type 1 or type 2 diabetes. Blood glucose on admission was measured in 893 (59\%). The number of patients triaged to each medical specialty, along with the number and proportion of individuals with diabetes in each specialty, is shown in Table 1 . The mean ages for the six blood glucose groups $<6.5 \mathrm{mmol} / \mathrm{l}, 6.5-7 \mathrm{mmol} / \mathrm{l}, 7.1-9 \mathrm{mmol} / \mathrm{l}$, 9.1-11 mmol/l, $11.1-20 \mathrm{mmol} / \mathrm{l}$ and $>20 \mathrm{mmol} / \mathrm{l}$ were 69.6 (standard deviation (SD) 18.8) years, 72.7 (15.5) years, 76.5 (13.9) years, 74.9 (13.7) years, 68.4 (17.3) years and $60.6(2.26)$ years, respectively.

Table 1. All admissions to the acute medical unit according to triaged specialty during February 2010 and the proportion with diabetes.

$\begin{array}{lll}\text { Specialty } & \text { All } & \begin{array}{c}\text { Number of patients } \\ \text { With diabetes (\%) }\end{array} \\ \text { Medicine for the elderly } & 577 & 94(16.3) \\ \text { Cardiology } & 221 & 25(11.3) \\ \text { Respiratory } & 200 & 28(14) \\ \text { Nephrology } & 30 & 9(30) \\ \text { Gastroenterology } & 132 & 18(13.6) \\ \text { Endocrinology } & 30 & 22(73) \\ \text { Neurology } & 77 & 12(16.9) \\ \text { Dermatology } & 1 & 0 \\ \text { Haematology } & 16 & 0 \\ \text { Oncology } & 56 & 4(7.4) \\ \text { General medicine } & 162 & 27(16.7)\end{array}$

Table 2. Patients within each blood glucose group on admission, length of stay for that admission, and readmissions and mortality within 28 days of discharge.

$\begin{array}{lllll}\begin{array}{l}\text { Blood } \\ \text { glucose } \\ \text { (mmol/I) }\end{array} & \begin{array}{l}\text { Number } \\ \text { of } \\ \text { patients }\end{array} & \begin{array}{l}\text { Mean } \\ \text { (median) } \\ \text { length of } \\ \text { stay (days) }\end{array} & \begin{array}{l}\text { Number } \\ \text { (\%) of } \\ \text { readmissions } \\ \text { within 28 days }\end{array} & \begin{array}{l}\text { Number (\%) } \\ \text { of deaths } \\ \text { within } \\ \mathbf{2 8} \text { days }\end{array} \\ <6.5 & 455 & 8.8(4) & 29(6.4) & 22(4.8) \\ 6.5-7 & 116 & 11.3(7) & 7(6.0) & 7(6.0) \\ 7.1-9 & 155 & 10.2(5) & 15(9.7) & 9(5.8) \\ 9.1-11 & 64 & 10.6(5) & 8(12.5) & 11(17.2) \\ 11.1-20 & 70 & 12(5.5) & 7(10.0) & 12(17.1) \\ >20 & 33 & 9.1(5) & 5(15.0) & 2(6.1)\end{array}$

Data for length of stay is shown in Table 2 by blood glucose group on admission. A significant difference in length of stay was seen for patients with blood glucose $>6.5 \mathrm{mmol} / \mathrm{l}$ on admission compared to those with blood glucose $<6.5 \mathrm{mmol} / 1$ on admission $(\mathrm{p}=0.002)$. The median length of stay was 6 days for those with blood glucose $>6.5 \mathrm{mmol} / \mathrm{l}$ on admission and 4 days for those with blood glucose $<6.5 \mathrm{mmol} / \mathrm{l}$ on admission, and the mean lengths of stay were 10.8 days and 8.8 days, respectively.

Table 2 also shows the 28-day readmission rates for each blood glucose group. Overall, 37 (2.5\%) of the total number of admissions were individuals without an existing diagnosis of diabetes but with blood glucose $\geqslant 11.1 \mathrm{mmol} / \mathrm{l}$ on admission. Of these, only 19 (51.4\%) received further follow up. Of those patients triaged to each specialty, the follow up rates were: $100 \%$ for endocrinology, $100 \%$ for general medicine (under the nephrology team allocation of the general medicine take), $53.8 \%$ for medicine for the elderly, 50\% for respiratory, 50\% for renal, $33.3 \%$ for cardiology and $0 \%$ each for gastroenterology, neurology and oncology. No patients without diagnosed diabetes but with blood glucose $\geqslant 11.1 \mathrm{mmol} / \mathrm{l}$ on admission were triaged to dermatology or haematology.

Table 2 also shows 28-day mortality. A statistically significant difference in mortality was seen as blood glucose on admission increased, except in those with the highest levels of blood glucose: for patients with blood glucose of 9.1-20 mmol/l on admission, 28 -day mortality was $17.2 \%$, which was significantly higher than the 28 -day mortality of $5.2 \%$ for those with blood glucose $\leqslant 9 \mathrm{mmol} / \mathrm{l}$ on admission $(\mathrm{p}<0.0001)$. The mean ages for these two groups were 70.2 years and 71.4 years, respectively, which suggests that age was unlikely to be a factor in this increased mortality.

\section{Discussion}

This study adds further evidence for dysglycaemia as an independent variable that affects the length of hospital stay and demonstrates that it can be used as a prognostic marker for a non-selected population with a range of medical problems admitted acutely to secondary care. However, despite the utility of blood glucose on admission, this study shows that it is measured for only $59 \%$ of admissions. Poor rates of checking blood glucose on admission have been seen in other studies and have been attributed to a low value being placed on its monitoring. ${ }^{6}$ However, this study adds weight to the argument for routine testing of blood glucose on admission on economic grounds due to the impact and saving that it can have through predictions of length of stay and short-term prognostication.

Short-term (28-day) readmission and mortality for all admissions generally increased with increasing levels of blood glucose on admission. Short-term readmission rates were highest for those with blood glucose $>20.1 \mathrm{mmol} / \mathrm{l}$ on admission, while 28 day mortality was highest for those with blood glucose levels on admission of 9.1-11 mmol/l and 11.1-20 mmol/l. Recognition of this trend allows prognostication and also demonstrates that 
improved glycaemic control is associated with better short-term mortality and readmission and should be targeted as part of the management of the primary pathology. ${ }^{7}$ Therapy aimed at correcting mild hyperglycaemia after stroke has also shown to improve neurological outcomes at 30 days. ${ }^{8}$

Of note was the decrease in 28-day mortality in the groups with the highest blood glucose levels on admission $(>20.1$ $\mathrm{mmol} / \mathrm{l})$. This likely represents patients with hyperglycaemic emergencies, such as diabetic ketoacidosis and hyperosmolar non-ketotic hyperglycaemia, which may have lower mortality due to the increased attention paid to their glucose levels and general physiological monitoring and treatment. Furthermore, this group had a lower mean average age than the other groups and so was more likely to have greater physiological reserve. Consequently, 28-day mortality was lower than in the groups with blood glucose of $9.1-11 \mathrm{mmol} / \mathrm{l}$ and $11.1-20 \mathrm{mmol} / \mathrm{l}$ on admission, in whom hyperglycaemia is likely to have represented a stress response rather than the primary pathology. ${ }^{9}$ Conversely, those people admitted with high blood glucose under other specialty teams are often looked after by junior doctors who have little or no knowledge of diabetes or confidence in its management, which may contribute to the longer length of stay and poorer outcomes. ${ }^{10}$

Despite the importance of the level of blood glucose on admission for length of hospital stay and short-term readmission and mortality and as a long-term risk factor for a range of diseases, this study demonstrates that the follow up of unexpected hyperglycaemia in individuals without an existing diagnosis of diabetes remains poor for most medical specialties. However, the results should be interpreted with some care, as, for example, patients admitted to the oncology service were typically beginning end-of-life care and therefore further investigation of hyperglycaemia would be inappropriate. Overall, these results reflect similar poor rates of follow up elsewhere. ${ }^{6}$

The authors recognise the limitations of this study, in that it was not possible to determine long-term glycaemic control by measuring the level of glycosylated haemoglobin $\left(\mathrm{HbA}_{1 c}\right)$ for each admission. Further study in this area would help determine whether the duration of hyperglycaemia affects length of hospitalisation and 28-day outcomes. Furthermore, we did not examine the relationship between mortality, glucose and age.

This study demonstrates the benefit of measuring blood glucose at the time of admission across a broad range of acute medical presentations. The results show that blood glucose on admission acts not only as an important factor in the length of hospital stay but also as a prognostic marker for short-term readmission rates and mortality, regardless of the presence of diabetes. At a time of increasing demand on hospital services, such information can help towards estimating bed occupancy and discharge planning. Furthermore, this study highlights that admission hyperglycaemia is an important variable and that,

\section{Key points}

High blood glucose levels in acutely ill medical patients admitted to hospital are associated with poor outcomes, as assessed by length of stay, 28-day readmission rates and 28-day mortality

Patients with the highest blood glucose levels on admission may be protected from these poor outcomes through specialist diabetes care

despite its ease of testing, it is frequently under-recognised and underinvestigated by admitting teams.

\section{References}

1 Umpierrez GE, Isaacs SD, Bazargan N et al. Hyperglycemia: An independent marker of in-hospital mortality in patients with undiagnosed diabetes. J Clin Endocrinol Metab 2002;87:978-82.

2 Eurich DT, Gamble JM, Marrie TJ et al. Dysglycaemia and 90 day and 1 year risks of death or readmission in patients hospitalised for community-acquired pneumonia. Diabetologia 2010;53:497-503.

3 Foo K, Cooper J, Deaner A et al. A single serum glucose measurement predicts adverse outcomes across the whole range of acute coronary syndromes. Heart 2003;89:512-6.

4 Williams LS, Rotich J, Qi R et al. Effects of admission hyperglycemia on mortality and costs in acute ischemic stroke. Neurology 2002;59:67-71.

5 Whitcomb BW, Pradhan EK, Pittas AG et al. Impact of admission hyperglycemia on hospital mortality in various intensive care unit populations. Crit Care Med 2005;33:2772-7.

6 Leite SAO, Locatelli SB, Niece SP et al. Impact of hyperglycemia on morbidity and mortality, length of hospitalization and rates of re-hospitalization in a general hospital setting in Brazil. Diabetol Metab Syndr 2010;2:49.

7 Chase JG, Shaw G, Le Compte A et al. Implementation and evaluation of the SPRINT protocol for tight glycaemic control in critically ill patients: a clinical practice change. Crit Care 2008;12:R49.

8 Straszewski J, Brodacki B, Kotowicz J et al. Intravenous insulin therapy in the maintenance of strict glycemic control in nondiabetic acute stroke patients with mild hyperglycemia. J Stroke Cerebrovasc Dis 2011;20:150-4.

9 Dungan KM, Braithwaite SS, Preiser J-C. Stress hyperglycaemia. Lancet 2009;373:1798-807.

10 George JT, Warriner D, McGrane DJ et al. Lack of confidence among trainee doctors in the management of diabetes: the Trainees Own Perception of Delivery of Care (TOPDOC) Diabetes Study. QJM 2011;104:761-6.

11 Timmer JR, Hoekstra M, Nijsten MW et al. Prognostic value of admission glycosylated hemoglobin and glucose in nondiabetic patients with ST-segment elevation myocardial infarction treated with percutaneous coronary intervention/clinical perspective. Circulation 2011;124:704-11.

Address for correspondence: Dr Ketan Dhatariya, Elsie Bertram Diabetes Centre, Norfolk and Norwich University Hospitals NHS Foundation Trust, Colney Lane, Norwich, NR4 7UY.

Email: ketan.dhatariya@nnuh.nhs.uk 